\title{
Effect of 1,8-Pyrenequinone, a Mutagenicity Enhancing Agent, on Metabolisms of 2-Acetylaminofluorene in Liver Microsomal Fraction
}

\author{
Hitoshi Oкамото, Daisuke Yoshida, Fujio Goto \\ and Hirohisa OMURA* \\ Central Research Institute, The Japan Tobacco \& Salt Public Corporation, \\ 6-2 Umegaoka, Midori-ku, Yokohama, Kanagawa 227, Japan \\ *Department of Food Science and Technology, Faculty of Agriculture, \\ Kyushu University, Fukuoka 812, Japan \\ Received February 5, 1982
}

\begin{abstract}
The addition of 1,8-pyrenequinone into the assay system containing rat liver homogenates ( $\mathrm{S}$ 9) caused an approximately 10-fold increase in the mutagenicity of 2-acetylaminofluorene (AAF) in the current Salmonella reversion assay system. Since no chemical reaction between 1,8-pyrenequinone and AAF was observed, the in vitro effects of 1,8-pyrenequinone on the metabolisms of AAF with S-9 mix were studied. The enhancement of mutagenicity by 1,8-pyrenequinone was not dependent on the dose of NADPH under the present assay condition. The mutagenicity of AAF was increased approximately 4 -fold by the addition of 1,8-pyrenequinone into microsomes, whereas it remained at the spontaneous level in the presence of cytosol. However, by reconstituting microsomes with cytosol, the mutagenicity enhancing activity was recovered to the original level. Since 1,8-pyrenequinone inhibited the AAF hydroxylase activity, chemical analysis of the incubation mixture of AAF was tried. This indicated that a higher amount of unmetabolized AAF remained and higher amounts of 2-aminofluorene and $N$-hydroxy-2-acetylaminofluorene were accumulated in the presence of 1,8-pyrenequinone compared with those in the absence of 1,8pyrenequinone. From these results, it seems probable that 1,8-pyrenequinone inhibits $C$ hydroxylation (the detoxifying pathway) and promotes $N$-hydroxylation (the activating pathway) as well as deacetylation in the AAF metabolism.
\end{abstract}

We previously reported that certain pyrolysis products of carbohydrates enhanced the mutagenicities of aromatic amines such as 2-acetylaminofluorene (AAF), 2-aminofluorene (AF), 2-aminoanthracene (AA) and Trp-P,* a potent mutagen detected in the tryptophan pyrolysate ${ }^{1)}$ and broiled fish cooked in the ordinary way. $\left.{ }^{2}\right)$ We also reported that pyrene, 1-methylpyrene and 1-hydroxypyrene were isolated from cellulose pyrolysates and identified as the substances enhancing the mutagenicity of AAF. ${ }^{3 \sim 5)}$ The enhancement of mutagenicity by these pyrenes was revealed only in the presence of rat liver homogenates (S-9). When pyrene was metabolized in the presence of S-9 mix, 1-hydroxypyrene, 4,5- dihydro-4,5-dihydroxypyrene, 1,6-dihydroxypyrene, 1,8-dihydroxypyrene, 1,6-pyrenequinone and 1,8-pyrenequinone were detected in the metabolites. ${ }^{6,7)}$ Among these metabolic products, 1,8-pyrenequinone showed the highest mutagenicity enhancing activity, and it was suggested that 1,8-pyrenequinone might play an important role in the exhibition of mutagenicity enhancing activity by pyrene.

We also reported that 1,8-pyrenequinone enhanced the mutagenicities of aromatic amines such as AAF, AF and AA, which usually require metabolic activation to reveal their mutagenicity. ${ }^{8)}$ However, the mutagenicities of the direct acting mutagens were not influenced by the addition of 1,8-pyrenequi-

* The mixture of Trp-P-1 (3-amino-1,4-dimethyl-5H-pyrido[4,3- $b]$ indole) and Trp-P-2 (3-amino-1-methyl-5Hpyrido[4,3- $b$ indole). 
none. ${ }^{8)}$

In this paper, the in vitro effects of $1,8-$ pyrenequinone as a modulator of the metabolism of AAF are studied and the mechanism for mutagenicity enhancing action of AAF by 1,8 -pyrenequinone is discussed.

\section{MATERIALS AND METHODS}

Chemicals. AAF, AF, 1,4-naphthoquinone, 2-methylnaphthoquinone, 2-methylanthraquinone and Vitamin $\mathrm{K}_{1}$ were of the purest grade available from commercial sources. 1,8-Pyrenequinone was synthesized by the method of Arnold et al. ${ }^{\text {9) }} \mathrm{N}$-Hydroxy-2-acetylaminofluorene (N-OH-AAF) was a generous gift from Dr. Y. Yamazoe, University of Keio, Tokyo. Ubiquinone 9 and ubiquinone 10 were generously supplied by Dr. T. Ikeda of this Institute. NADPH and glucose-6-phosphate (G-6-P) were purchased from Oriental Yeast Company Ltd., Tokyo.

Preparation of S-9, microsomes and cytosol. The S-9 fraction was prepared from the livers of $200 \mathrm{~g}$ male Sprague-Dawley rats induced 5 days prior to sacrifice by a single injection of polychlorinated biphenyl according to the method of Ames et al. ${ }^{10)}$ The microsomes and cytosol were prepared by centrifugation of the S-9 fraction at $105,000 \times g$ for $1 \mathrm{hr}$ at $4^{\circ} \mathrm{C}$. The supernatant and the pellet fraction were cytosol and microsomes respectively, and the latter was resuspended in cold $0.15 \mathrm{M} \mathrm{KCl}$ of the same volume as that of the original S-9. For the mutagenicity assay, cofactors such as NADPH and G-6-P were incorporated in the respective enzyme fractions.

Determination of AAF hydroxylase activity. The hydroxylase activity of AAF was determined according to the method of Cramer et $a l .{ }^{11)}$ The reaction mixture for assay contained $24 \mu \mathrm{mol}$ of $\mathrm{MgCl}_{2}, 100 \mu \mathrm{mol}$ of $\mathrm{KCl}, 300$ $\mu \mathrm{mol}$ of sodium phosphate buffer, $\mathrm{pH} 7.4,10.44 \mu \mathrm{mol}$ of NADPH, $13.44 \mu \mathrm{mol}$ of G-6-P, $0.3 \mathrm{ml}$ of S-9 and 0.23 $\mu \mathrm{mol}$ of AAF in $50 \mu \mathrm{l}$ methanol with 0 to $0.86 \mu \mathrm{mol}$ of 1,8 pyrenequinone in $0.2 \mathrm{ml}$ methanol at the maximum. The total volume was between 3 and $3.25 \mathrm{ml}$. The mixtures were incubated in a shaker for various times at $37^{\circ} \mathrm{C}$ in a flask open to the air and the reaction was terminated by the addition of $1 \mathrm{ml}$ of $38 \% \mathrm{KOH}$. Ten minutes later the neutral and basic compounds were extracted into $20 \mathrm{ml}$ of a 1:1 mixture of diethyl ether and petroleum ether by vigorous shaking for $1 \mathrm{~min}$, and the optical density of the organic phase was determined at $288 \mathrm{~nm}$ in a spectrophotometer.

Preparation of incubation mixture for chemical analysis. The incubation mixture contained $40 \mu \mathrm{mol} \mathrm{MgCl}_{2}, 168$ $\mu \mathrm{mol} \mathrm{KCl}, 17.4 \mu \mathrm{mol} \mathrm{NADPH}, 22.4 \mu \mathrm{mol} \mathrm{G}-6-\mathrm{P}, 0.5$ mmol sodium phosphate buffer, $\mathrm{pH} 7.4,0.5 \mathrm{ml}$ microsomes and $0.45 \mu \mathrm{mol} \mathrm{AAF}$ in $0.1 \mathrm{ml}$ methanol with (B) or without (A) $0.43 \mu \mathrm{mol} 1,8$-pyrenequinone in $0.2 \mathrm{ml}$ methanol. The total volume was $5.1 \mathrm{ml}$ or $5.3 \mathrm{ml}$ and each reaction mixture was incubated for $30 \mathrm{~min}$ at $37^{\circ} \mathrm{C}$ in a flask open to the air.

Analysis of $A A F$ and $A F$. The reaction mixture was extracted 3 times with approximately $40 \mathrm{ml}$ of ether. The ether extracts were combined, evaporated to dryness and dissolved in $2 \mathrm{ml}$ of methanol for analysis by high-pressure liquid chromatography (HPLC). HPLC was carried out using a JASCO Tri Rotar chromatograph connected with a JASCO UVIDEC-100-II spectrophotometer and a JASCO FP550 spectrofluorometer. A $25 \mathrm{~cm}$ Zorbax ODS column maintained at $50^{\circ} \mathrm{C}$ was eluted using $65 \%$ methanol in water at a flow rate of $1.5 \mathrm{ml} / \mathrm{min}$. AAF was monitored by UV absorption at $280 \mathrm{~nm}$. AF was detected by spectrofluorometer at an excitation wavelength (EX) of $290 \mathrm{~nm}$ and at an emission wavelength (EM) of $370 \mathrm{~nm}$. The peak area of AAF was calculated by a Takeda Riken TR-2213 Integrator and for AF was calculated by a Shimadzu Chromatopac C-R1A. The AAF and AF contents of the sample solution were calculated from the calibration with authentic samples.

Analysis of $\mathrm{N}-\mathrm{OH}-\mathrm{AAF}$. N-OH-AAF was analyzed according to the procedure described by Hinson et al. ${ }^{12)}$ The reaction was terminated by addition of $5 \mathrm{ml}$ of acetone. The precipitated protein was separated by centrifugation, and the supernatant phase was decanted and extracted twice with approximately $40 \mathrm{ml}$ of ether. The ether extracts were combined and evaporated to dryness. The residues were then dissolved in $2 \mathrm{ml}$ of methanol and a sample of each was analyzed by HPLC. The HPLC assay was performed in a Shimadzu LC-3A chromatograph connected with a reverse-phase $\mathrm{C}_{18} \mu$ Bondapak column (3.9 $\times$ $300 \mathrm{~mm})$ and a Shimadzu spectrophotometric detector SPD-1 at $535 \mathrm{~nm}$. The carrier solvent consisted of $1 \mathrm{~mm}$ ferric chloride, $0.005 \mathrm{M}$ heptane sulfonic acid and $1 \%$ acetic acid dissolved in $70 \%$ methanol in water. Peak area was calculated by a Takeda Riken TR-2213 Integrator. The N$\mathrm{OH}-\mathrm{AAF}$ content of the sample solution was calculated from the calibration with authentic sample.

Recovery. The methanol solutions of the authentic AAF, AF and N-OH-AAF were treated using the same procedure as that for the analytical sample. The percentages of AAF, AF and N-OH-AAF recovered by this method were $96.2( \pm 1.2) \%, 86.1( \pm 1.1) \%$ and 86.6 $( \pm 3.6) \%$, respectively, from experiments repeated three times. The data indicated were corrected for the recovery.

Mutagenicity assay. Mutagenicity assays with Salmonella typhimurium TA98 were carried out using the standard plate test according to the method of Ames et $a l .{ }^{10)}$ as previously described. ${ }^{3)}$ To a test tube containing 
$2 \mathrm{ml}$ of molten top agar $(0.6 \%$ agar, $0.6 \% \mathrm{NaCl}, 0.1 \mu \mathrm{mol}$ histidine, $0.1 \mu \mathrm{mol}$ biotin) were added $0.1 \mathrm{ml}$ of the tester strain, $3 \mu \mathrm{g}$ of AAF, the sample suitably diluted in dimethyl sulfoxide, and $0.5 \mathrm{ml}$ of S-9 mix that contained $0.05 \mathrm{ml}$ of S-9, $4 \mu \mathrm{mol} \mathrm{MgCl}_{2}, 16.8 \mu \mathrm{mol} \mathrm{KCl}, 1.74 \mu \mathrm{mol}$ NADPH, $2.24 \mu \mathrm{mol} \mathrm{G}-6-\mathrm{P}$ and $50 \mu \mathrm{mol}$ sodium phosphate buffer, $\mathrm{pH}$ 7.4. The tube contents were mixed and poured onto an agar over layer. The number of histidine revertant colonies per plate was counted after incubation for 2 days at $37^{\circ} \mathrm{C}$ and the results given are expressed as the average number of $\mathrm{His}^{+}$colonies from duplicate plates.

\section{RESULTS}

Mutagenicity enhancing action of $A A F$ by 1,8 pyrenequinone

The mutagenicity of AAF assayed with the addition of different amounts of 1,8-pyrene-

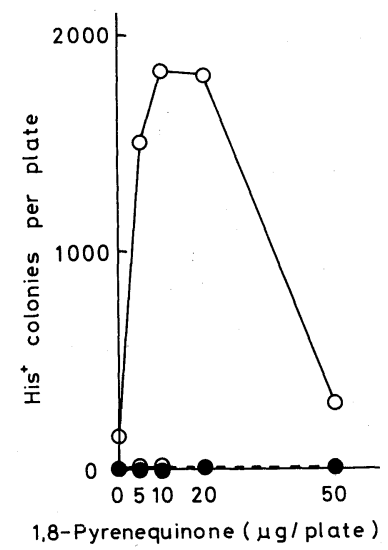

FIG. 1. Mutagenicity of the Mixture of 1,8-Pyrenequinone and AAF in the Presence or Absence of S-9 Mix. The mutagenicity of 1,8-pyrenequinone with $(O)$ or without (O) AAF was assayed using $S$. typhimurium TA98 in the absence (---) or presence $(-)$ of S-9 mix. quinone is shown in Fig. 1. The number of $\mathrm{His}^{+}$colonies in $3 \mu \mathrm{g}$ of AAF per plate was 170. The addition of 5 to $20 \mu \mathrm{g}$ of 1,8-pyrenequinone resulted in a 9- to 11-fold increase in the mutagenicity of AAF, but further increase in the amount of 1,8-pyrenequinone resulted in a decrease in the number of $\mathrm{His}^{+}$colonies. 1,8Pyrenequinone itself was not mutagenic toward S. typhimurium TA98.

\section{Chemical reaction between $A A F$ and 1,8- pyrenequinone}

The mixed solution without S-9 mix, which contained AAF and 1,8-pyrenequinone, was shaken in a water bath maintained at $37^{\circ} \mathrm{C}$. After 30 minutes an aliquot of the incubation mixture was subjected to thin-layer chromatography (TLC) and HPLC. Although the data are not shown, no incidence of any substances apart from AAF and 1,8-pyrenequinone was observed on analysis by TLC, and the amount of AAF eluted at the same retention time as the authentic AAF was unchanged on analysis by HPLC. This shows that a chemical reaction between AAF and 1,8-pyrenequinone did not occur in the solution and that 1,8-pyrenequinone would be comparatively stable in the assay medium.

Effect of 1,8-pyrenequinone on NADPH in S-9 mix

Quinones, particularly ubiquinones, play an important role as mediators of the electron transport from NADPH contained in S-9 mix. $^{13,14)} 1,8$-Pyrenequinone may act as an electron acceptor from NADPH in S-9 mix.

Table I. Effect of NADPH on the Mutagenicity Enhancement by 1,8-Pyrenequinone

\begin{tabular}{|c|c|c|c|c|}
\hline \multirow{2}{*}{$\begin{array}{c}\text { NADPH } \\
(\mu \mathrm{mol} / \text { plate })\end{array}$} & \multicolumn{4}{|c|}{$\mathrm{His}^{+}$colonies per plate ${ }^{a}$} \\
\hline & Spontaneous & $\begin{array}{l}\text { AAF } \\
(3 \mu \mathrm{g})\end{array}$ & $\begin{array}{l}\text { 1,8-Pyrenequinone } \\
\qquad(10 \mu \mathrm{g})\end{array}$ & $\underset{\text { 1,8-Pyrenequinone }}{\mathrm{AAF}+}$ \\
\hline 0 & 24 & 41 & 18 & 68 \\
\hline 1.74 & 39 & 182 & 51 & 1098 \\
\hline 3.48 & 47 & 179 & 50 & 1196 \\
\hline 5.22 & 36 & 192 & 60 & 1215 \\
\hline 8.70 & 44 & 197 & 49 & 1037 \\
\hline
\end{tabular}

a S. typhimurium TA98. 
Consequently the mutagenicities of AAF with 1,8-pyrenequinone at various concentrations of NADPH and subsequently the effect of various quinones on the mutagenicity of AAF were firstly determined. As shown in Table I, the mutagenicity of the mixture of AAF and 1,8-pyrenequinone hardly changed with the addition of NADPH up to 5 times of the usual level $(1.74 \mu \mathrm{mol})$. In the absence of NADPH, the mutagenicity of AAF was not enhanced at all by the addition of 1,8-pyrenequinone. This suggests that NADPH is required for revealing the enhancement of mutagenicity, and that the mutagenicity enhancement by 1,8-pyrenequinone is not influenced by the amount of NADPH used in this assay condition.

Subsequent experiments examining the effect of various quinones on the mutagenicity of AAF were carried out. As shown in Table II, ubiquinone 9 and ubiquinone 10 did not enhance the mutagenicity of AAF. Naphthoquinone and its methyl derivative, and Vitamin $K_{1}$ also enhanced little the mutagenicity of AAF. 2-Methylanthraquinone resulted in a 2- to 3-fold increase in the number of $\mathrm{His}^{+}$colonies of AAF, although the degree of enhancement was much lower than that of 1,8-pyrenequinone. These results indicate that the enhancing action of mutagenicity may not be common for quinone compounds but it would be rather specific to 1,8-pyrenequinone.

\section{Effect of 1,8-pyrenequinone on the mutagenicity} of AAF in microsomes and cytosol

From the above results, it would seem that the mutagenicity enhancement of AAF is exhibited by the interaction between 1,8-pyrenequinone and S-9 mix. Therefore, the effects of 1,8-pyrenequinone on the mutagenicity of AAF were examined as to microsomes, cytosol, the reconstituted mixture of microsomes and cytosol, and S-9. As shown in Fig. 2, the mutagenicity of AAF was increased approximately 4-fold by the addition of $10 \mu \mathrm{g}$ of $1,8-$ pyrenequinone in the presence of microsomes, whereas it remained only at the spontaneous level in the presence of cytosol. The addition of microsomes in combination with cytosol to the assay medium markedly increased the mutagenicity of AAF with 1,8-pyrenequinone, which was approximately recovered at a level similar to the orginal S-9. These facts show that

TABLE II. EFFECT OF VARIOUS QuinONES ON MUTAGENicity OF AAF

\begin{tabular}{|c|c|c|c|c|}
\hline \multirow[b]{2}{*}{ Experiment } & \multirow[b]{2}{*}{ Quinone } & \multirow{2}{*}{$\begin{array}{c}\text { Dose } \\
(\mu \mathrm{g} / \text { plate })\end{array}$} & \multicolumn{2}{|c|}{$\mathrm{His}^{+}$colonies per plate ${ }^{a}$} \\
\hline & & & $\begin{array}{c}\text { Without } \\
\text { AAF }\end{array}$ & $\begin{array}{l}\text { With AAF } \\
(3 \mu \mathrm{g} / \text { plate })\end{array}$ \\
\hline \multirow{5}{*}{1} & None & - & 41 & 158 \\
\hline & Ubiquinone 9 & 5 & 53 & 181 \\
\hline & & 10 & 48 & 162 \\
\hline & Ubiquinone 10 & 5 & 53 & 166 \\
\hline & & 10 & 37 & 191 \\
\hline \multirow{11}{*}{2} & None & - & 30 & 173 \\
\hline & 1,4-Naphthoquinone & 5 & 34 & 209 \\
\hline & & 10 & 37 & 224 \\
\hline & & 50 & 31 & 221 \\
\hline & 2-Methylnaphthoquinone & 5 & 48 & 226 \\
\hline & & 10 & 34 & 210 \\
\hline & 2-Methylanthraquinone & 5 & 24 & 373 \\
\hline & & 10 & 29 & 496 \\
\hline & Vitamin $\mathrm{K}_{1}$ & 5 & 33 & 184 \\
\hline & & 10 & 35 & 171 \\
\hline & & 50 & 30 & 222 \\
\hline
\end{tabular}




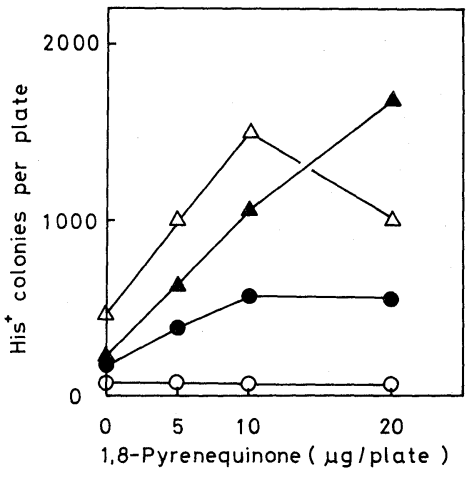

FIG. 2. Effect of 1,8-Pyrenequinone on the Mutagenicity of AAF in the Presence of Microsomes or Cytosol.

The mutagenicity of the mixture of $\mathrm{AAF}(3 \mu \mathrm{g})$ and $1,8-$ pyrenequinone was determined in the presence of microsomes (-O), cytosol ( $-\mathrm{O}-\mathrm{O})$, S-9 $(\mathbf{\Delta}-\mathbf{\Delta})$ and both microsomes and cytosol $(\triangle-\triangle)$.

cytosol plays a role together with microsomes in the mutagenicity enhancement of AAF by 1,8-pyrenequinone.

\section{Effect of 1,8-pyrenequinone on hydroxylation of} $A A F$

The activity of hydroxylation which is the first step of AAF metabolism was determined. The effect of incubation time on the hydroxylation of AAF by rat liver homogenates is shown in Fig. 3. Approximately $93 \%$ of AAF was converted to phenolic metabolites upon incubation for $30 \mathrm{~min}$. The effect of 1,8pyrenequinone on the hydroxylation of AAF is shown in Fig. 4. The incubation time with $\mathrm{S}$ $9 \mathrm{mix}$ was $10 \mathrm{~min}$. At equimolar levels, 1,8pyrenequinone inhibited about $50 \%$ of the hydroxylation activity of AAF. The addition of $0.86 \mu \mathrm{mol}$ of 1,8 -pyrenequinone inhibited about $90 \%$ of the activity.

\section{Determination of the metabolites of $A A F$ with} or without 1,8-pyrenequinone

The AAF, AF and N-OH-AAF contents contained in the incubation mixture of AAF with (sample B) or without (sample A) 1,8pyrenequinone are shown in Table III. The amount of AAF detected in sample A corresponded to $1.6 \%$ of the original AAF, whereas the amount of AAF detected in sam-

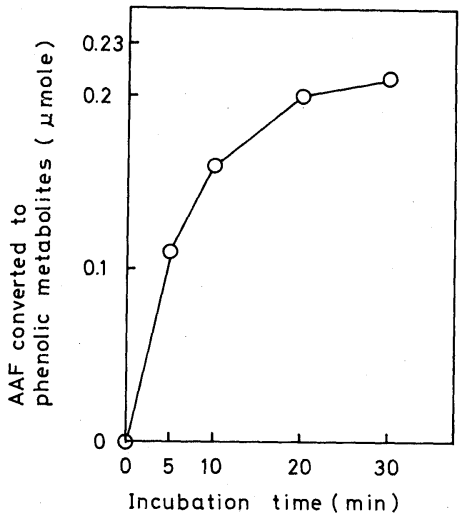

Fig. 3. Effect of the Incubation Time on Hydroxylation of AAF by S-9 Mix.

Aliquots of individual mixtures containing $0.23 \mu \mathrm{mol}$ of AAF were incubated at $37^{\circ} \mathrm{C}$ and extracted into $20 \mathrm{ml}$ of a $1: 1$ mixture of petroleum ether and diethyl ether. The optical density of the organic phase was determined at $288 \mathrm{~nm}$ in a spectrophotometer.

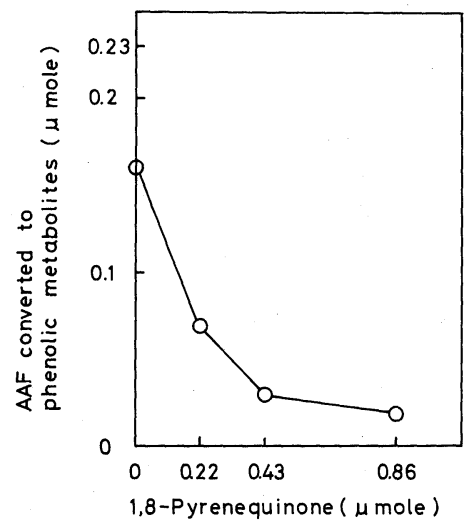

FIG. 4. Effect of 1,8-Pyrenequinone on Hydroxylation of AAF by S-9 Mix.

The mixtures containing $0.23 \mu \mathrm{mol}$ of $\mathrm{AAF}$ with or without 1,8 -pyrenequinone were incubated at $37^{\circ} \mathrm{C}$ for 10 min. For details, see the legend of Fig. 3.

TABle III. Determination of the Metabolites OF AAF WITH OR WITHOUT 1,8-PYRENEQUINONE

\begin{tabular}{lrcr} 
& \multicolumn{2}{c}{$\begin{array}{c}\text { Metabolic product } \\
\text { Snmol) }\end{array}$} & AAF \\
\cline { 2 - 3 } & AF & N-OH-AAF & (nmol) \\
\cline { 2 - 3 } & 2.9 & 5.8 & 7.1 \\
$\begin{array}{l}\text { AAF } \\
\text { AAF +1,8- } \\
\text { Pyrenequinone }\end{array}$ & 43.4 & 42.3 & 179.6 \\
\hline
\end{tabular}

Substrate: AAF, 450 nmol; 1,8-pyrenequinone, $430 \mathrm{nmol}$. 
ple B corresponded to about $40 \%$ of the original AAF. This shows that the original AAF was mostly metabolized in the absence of 1,8-pyrenequinone while it remained relatively unmetabolized in the presence of 1,8-pyrenequinone.

The addition of 1,8-pyrenequinone caused about a 15-fold increase in the formation of AF from AAF. This shows that 1,8-pyrenequinone promoted the deacetylation of AAF.

The addition of 1,8-pyrenequinone caused about a 7-fold increase in the formation of $\mathrm{N}$ $\mathrm{OH}-\mathrm{AAF}$ from AAF. This shows that the addition of 1,8-pyrenequinone to rat liver microsomes stimulates the $N$-hydroxylation of AAF.

Effect of 1,8-pyrenequinone on the mutagenicity of $\mathrm{N}-\mathrm{OH}-\mathrm{AAF}$ in microsomes and cytosol The effect of 1,8-pyrenequinone on the mu-

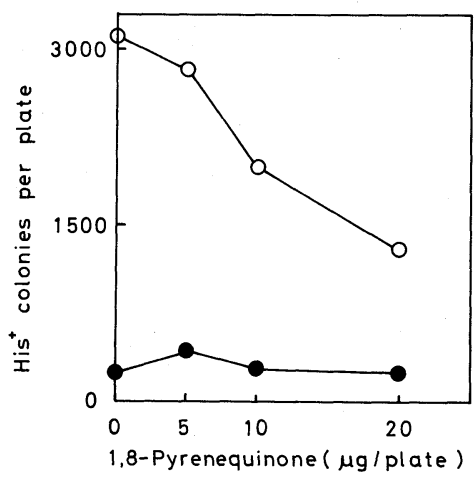

FIG. 5. Effect of 1,8-Pyrenequinone on Mutagenicity of N-OH-AAF in the Presence of Microsomes or Cytosol.

The mutagenicity of the mixture of N-OH-AAF $(1 \mu \mathrm{g})$ and 1,8-pyrenequinone was determined in the presence of microsomes (-O) and cytosol $(\mathrm{O}-\mathrm{O})$. tagenicity of $\mathrm{N}-\mathrm{OH}-\mathrm{AAF}$ in microsomes or cytosol is shown in Fig. 5. N-OH-AAF showed higher mutagenic activity in the presence of cytosol than in the presence of microsomes. The mutagenicity of $\mathrm{N}-\mathrm{OH}-\mathrm{AAF}$ decreased almost linearly with the dose level of 1,8pyrenequinone added in the presence of cytosol. However, approximately $40 \%$ of the mutagenic activity still remained with the addition of $20 \mu \mathrm{g}$ of 1,8-pyrenequinone. On the other hand, there was little influence of 1,8pyrenequinone on the mutagenicity of $\mathrm{N}-\mathrm{OH}$ $\mathrm{AAF}$ in the presence of microsomes.

\section{DISCUSSION}

A number of studies on the metabolism of AAF have provided important insights into the mechanism of chemical carcinogenesis. $\mathrm{N}$ Hydroxylation is a rate-limiting step in the activation of AAF with subsequent deacetylation and this is the most important metabolic pathway in the activation of $\mathrm{N}-\mathrm{OH}-\mathrm{AAF}$ to form direct mutagens. Formation of a potent mutagen from $\mathrm{N}-\mathrm{OH}-\mathrm{AAF}$ by activation with a rat liver cytosolic fraction is shown in the Salmonella reversion system. ${ }^{15 \sim 18)}$ This was also confirmed by our experiments (Fig. 5). Presumably the mutagenic species formed is $N$-hydroxy-2-aminofluorene ( $\mathrm{N}-\mathrm{OH}-\mathrm{AF}$ ), which is considered to be the ultimate mutagen. ${ }^{15,16,19)}$ The main metabolic pathway of $\mathrm{AAF}$ and the working points of 1,8-pyrenequinone are summarized in Fig. 6.

About $40 \%$ of AAF remained unmetabolized in the incubation mixture of AAF by the

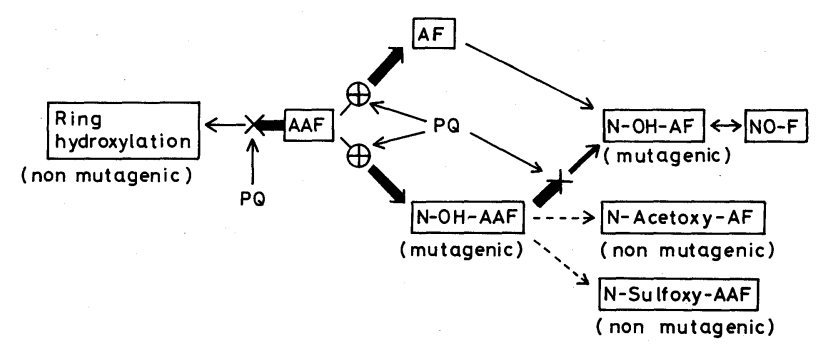

FIG. 6. Proposed Mechanism for the Mutagenic Activation of AAF with 1,8-Pyrenequinone.

The bold lines indicate the main pathway. NO-F, nitrosofluorene; $\mathrm{PQ}, 1,8$-pyrenequinone; $\times$, inhibition by 1,8-pyrenequinone; $\otimes$, promotion by 1,8 -pyrenequinone. 
addition of 1,8-pyrenequinone (Table III). This was also supported by the fact that $1,8-$ pyrenequinone inhibited hydroxylation which is considered as the first step in the metabolism of AAF (Fig. 4). However, 1,8-pyrenequinone stimulated the metabolism of AAF to $\mathrm{N}-\mathrm{OH}-\mathrm{AAF}$ which is a weakly mutagenic intermediate of AAF (Table III). These facts demonstrate that 1,8-pyrenequinone do not inhibit the $N$-hydroxylation, i.e., activating pathway, but inhibits the $C$-hydroxylation (ring-hydroxylation) of AAF, i.e., detoxifying pathway.

1,8-Pyrenequinone inhibited the enzyme activity of cytosol which mediates the metabolism of N-OH-AAF to further mutagenic species (Fig. 5). However, cytosol maintained enough mutagenic potency to reveal the mutagenicity of N-OH-AAF (Fig. 5). As shown in Fig. 1, the addition up to $20 \mu \mathrm{g}$ of 1,8 -pyrenequinone to the assay medium enhanced the mutagenicity of AAF, but the addition of $50 \mu \mathrm{g}$ of 1,8-pyrenequinone resulted in a decrease of mutagenicity. This may be due to an inhibition of cytosolic activity by the addition of $50 \mu \mathrm{g}$ 1,8-pyrenequinone.

When 1,8-pyrenequinone was added to the incubation mixture, a higher amount of AF was detected (Table III). Further metabolism of AF formed by microsomal enzymes may also produce N-OH-AF by the subsequent $N$ hydroxylation, whereas, as shown in Fig. 2, the mutagenicity of AAF showed only a few fold increase with the addition of 1,8-pyrenequinone in the presence of microsomes. Therefore, it seems likely that the metabolism of AAF to N-OH-AF through AF may not be the main pathway.

The mechanism of enhancing action on the mutagenicity of AAF by 1,8-pyrenequinone may be speculated as follows. In the metabolism of AAF, 1,8-pyrenequinone would inhibit the detoxification process ( $C$-hydroxylation) and promote the activation process ( $N$-hydroxylation). In consequence, $\mathrm{N}-\mathrm{OH}$ -
AAF, a weak mutagenic intermediate, would be accumulated and then activated by the cytosolic fraction to form the ultimate mutagen, $\mathrm{N}-\mathrm{OH}-\mathrm{AF}$.

Acknowledgment. We thank Dr. Y. Yamazoe (Keio University, Tokyo) for his generous gift of $\mathrm{N}-\mathrm{OH}-\mathrm{AAF}$ and his valuable information on the analysis of it.

\section{REFERENCES}

1) T. Sugimura, T. Kawachi, M. Nagao, T. Yahagi, Y. Seino, T. Okamoto, K. Shudo, T. Kosuge, K. Tsuji, K. Wakabayashi, T. Iitaka and A. Itai, Proc. Jpn. Acad., 53, 58 (1977).

2) Z. Yamaizumi, T. Shiomi, H. Kasai, S. Nishimura, Y. Takahashi, M. Nagao and T. Sugimura, Cancer Letters, 9, 75 (1980).

3) H. Okamoto, S. Mizusaki, D. Yoshida and T. Matsumoto, Agric. Biol. Chem., 43, 1433 (1979).

4) H. Okamoto, D. Yoshida, T. Matsumoto and S. Mizusaki, Agric. Biol. Chem., 44, 563 (1980).

5) H. Okamoto and D. Yoshida, Agric. Biol. Chem., 45, 1291 (1981).

6) H. Okamoto and D. Yoshida, Cancer Letters, 11, 215 (1981).

7) H. Okamoto and D. Yoshida, Cancer Letters, 12, 323 (1981).

8) H. Okamoto and D. Yoshida, Mutation Res., 73, 203 (1980).

9) R. T. Arnold and R. Larson, J. Org. Chem., 5, 250 (1940).

10) B. N. Ames, J. McCann and E. Yamasaki, Mutation Res., 31, 347 (1975).

11) J. W. Cramer, J. A. Miller and E. C. Miller, J. Biol. Chem., 235, 250 (1960).

12) J. A. Hinson, L. R. Pohl and J. R. Gillette, Anal. Biochem., 101, 462 (1980).

13) M. J. Gibian and J. A. Rynd, Biochem. Biophys. Res. Commun., 34, 594 (1969).

14) A. Kröger and M. Klingenberg, Eur. J. Biochem., 34, 358 (1973).

15) D. L. Stout, J. N. Baptist, T. S. Matney and D. R. Shaw, Cancer Letters, 1, 269 (1976).

16) G. J. Mulder, J. A. Hinson, W. L. Nelson and S. S. Thorgeirsson, Biochem. Pharmacol., 26, 1356 (1977).

17) S. Sakai, C. E. Reinhold, P. J. Wirth and S. S. Thorgeirsson, Cancer Res., 38, 2058 (1978).

18) C. E. Weeks, W. T. Allaben, S. C. Louis, E. J. Lazear and C. M. King, Cancer Res., 38, 613 (1978).

19) W. E. Durston and B. N. Ames, Proc. Natl. Acad. Sci. U.S.A., 71, 737 (1974). 